\title{
Orientation of luminescent excitons in layered nanomaterials
}

\author{
Jon A. Schuller ${ }^{1,2,3 \star}$, Sinan Karaveli ${ }^{1}$, Theanne Schiros ${ }^{2}$, Keliang He ${ }^{4}$, Shyuan Yang ${ }^{2}$, loannis Kymissis ${ }^{2}$, \\ Jie Shan ${ }^{4}$ and Rashid Zia ${ }^{1 \star}$
}

In nanomaterials, optical anisotropies reveal a fundamental relationship between structural and optical properties ${ }^{1-6}$. Directional optical properties can be exploited to enhance the performance of optoelectronic devices ${ }^{7-9}$, optomechanical actuators $^{10}$ and metamaterials ${ }^{11}$. In layered materials, optical anisotropies may result from in-plane and out-of-plane dipoles associated with intra- and interlayer excitations, respectively. Here, we resolve the orientation of luminescent excitons and isolate photoluminescence signatures arising from distinct intra- and interlayer optical transitions. Combining analytical calculations with energy- and momentum-resolved spectroscopy, we distinguish between in-plane and out-of-plane oriented excitons in materials with weak or strong interlayer coupling- $\mathrm{MoS}_{2}$ and 3,4,9,10-perylene tetracarboxylic dianhydride (PTCDA), respectively. We demonstrate that photoluminescence from $\mathrm{MoS}_{2}$ mono-, bi- and trilayers originates solely from in-plane excitons, whereas PTCDA supports distinct in-plane and out-of-plane exciton species with different spectra, dipole strengths and temporal dynamics. The insights provided by this work are important for understanding fundamental excitonic properties in nanomaterials and designing optical systems that efficiently excite and collect light from exciton species with different orientations.

Different dipole orientations can be resolved by their distinct antenna-like radiation patterns (Fig. 1a). These radiation patterns can be discerned experimentally using back focal plane imaging ${ }^{12}$, where every point in the image plane corresponds to a distinct angle of emission, or in-plane photon momentum $k_{\|}=k_{0} n \sin (\theta)$. Momentum-resolved measurements have been used to determine the orientation of chromophores ${ }^{12,13}$ and to image radiation patterns of optical antennas ${ }^{8,14}$. By focusing back focal plane images onto the entrance slit of an imaging spectrograph, one can simultaneously decompose photoluminescence by photon momentum and energy (that is, frequency $\omega$ ). Energy-momentum spectroscopy has recently been used to distinguish and quantify the isotropic electric and magnetic dipole transitions in rare earth ions ${ }^{15}$.

Expanding the analytical framework of ref. 15 to account for emission within uniaxial media (Supplementary Section I), experimentally measured $s$ - and $p$-polarized photoluminescence counts, $N^{s, p}\left(\omega, k_{\|}\right)$, can be fit to a superposition of in-plane (IP) and out-of-plane (OP) dipole emission:

$$
\begin{aligned}
N^{s, p}\left(\omega, k_{\|}\right)= & C_{\text {exp }} C_{0}(\omega)\left[\tilde{\rho}_{\mathrm{IP}}^{s, p}\left(\omega, k_{\|}\right) \bar{n}_{\mathrm{IP}}\left|\mu_{\mathrm{IP}}(\omega)\right|^{2}\right. \\
& \left.+\tilde{\rho}_{\mathrm{OP}}^{s, p}\left(\omega, k_{\|}\right) \bar{n}_{\mathrm{OP}}\left|\mu_{\mathrm{OP}}(\omega)\right|^{2}\right]
\end{aligned}
$$

where $C_{\exp }$ is a proportionality constant that depends on experimental parameters (for example, excitation intensity and integration time), $C_{0}(\omega)$ is a sample and orientation-independent prefactor, $\tilde{\rho}_{\mathrm{IP}}^{s, p}\left(\omega, k_{\|}\right)$and $\tilde{\rho}_{\mathrm{OP}}^{s, p}\left(\omega, k_{\|}\right)$are the normalized local density of optical states (LDOS) for IP and OP dipoles, $\bar{n}_{\mathrm{IP}}$ and $\bar{n}_{\mathrm{OP}}$ are the time-averaged populations of IP and OP excitons, and $\mu_{\mathrm{IP}}(\omega)$ and $\mu_{\mathrm{OP}}(\omega)$ are the IP and OP dipole moments of a single emitter. Figure $1 \mathrm{~b}$ presents a schematic of the geometry considered in the calculations and experiments. We consider photoluminescence emitted in the $y-z$ plane, such that $k_{\|}=k_{y}$. We collect $s$-polarized light using an $x$-oriented polarizer, and $p$-polarized light using a $y$-oriented polarizer. Figure $1 \mathrm{c}$ shows the calculated LDOS for IP- (blue) and OP-oriented (red) dipoles emitting at $700 \mathrm{~nm}$, located in the middle of a 20-nm-thin uniaxial layer of PTCDA with IP and OP refractive indices of $n_{\mathrm{o}}=2.1$ and $n_{\mathrm{e}}=1.6$, respectively, sitting on a quartz substrate $(n=1.5)$. The $s$-polarized light emission arises solely from IP dipoles (that is, $\left.\tilde{\rho}_{\mathrm{OP}}^{s, p}\left(\omega, k_{\|}\right)=0\right)$, but $p$-polarized light can contain contributions from both IP and OP dipoles. The significant difference in angular dependence allows us to decompose $p$-polarized spectra into relative contributions from the IP and OP dipoles.

Important conclusions can be drawn from a close examination of Fig. 1c. First, OP dipoles emit very little light into momentum states that are collected by optical systems with a moderate numerical aperture. By integrating the LDOS in the two-dimensional $k_{x}-k_{y}$ plane, we can determine the amount of photoluminescence collected by an optical system with a particular numerical aperture. For example, of the total light emitted into the substrate by OP dipoles $\left(\left|k_{\|} / k_{0}\right|<1.5\right)$, less than $5 \%$ falls within the angular range collected by a numerical aperture of $0.8\left(\left|k_{\|} / k_{0}\right|<0.8\right)$. To study the behaviour of OP excitons, the use of high-numerical-aperture collection optics is imperative. Second, the IP and OP LDOS differ significantly at the onset of total internal reflection at the air interface, $k_{\|}=k_{0}$. At this critical momentum, IP dipole emission vanishes, while OP dipole emission is maximized. Thus, the relative amount of photoluminescence collected from IP and OP dipoles strongly depends on the experimental geometry. For this reason, we use a combination of LDOS calculations and momentumresolved photoluminescence measurements to infer relative measures of the net IP and OP dipole strengths $\bar{n}_{\mathrm{IP}}\left|\mu_{\mathrm{IP}}(\omega)\right|^{2}$ and $\bar{n}_{\mathrm{OP}}\left|\mu_{\mathrm{OP}}(\omega)\right|^{2}$. Although we cannot determine the absolute dipole strengths, we can determine how they vary relative to wavelength and dipole orientation-properties that do not depend on the specific experimental geometry.

Recently, researchers discovered that monolayers of $\mathrm{MoS}_{2}$ exhibit a direct energy gap with a significantly enhanced photoluminescence quantum yield compared with indirect-gap bulk samples ${ }^{16,17}$. As a perfectly oriented, highly anisotropic material with measurable photoluminescence, $\mathrm{MoS}_{2}$ is an ideal system for

'School of Engineering, Brown University, Providence, Rhode Island 02912, USA, Energy Frontier Research Center, Columbia University, New York, New York 10027, USA, ${ }^{3}$ Department of Electrical and Computer Engineering, University of California, Santa Barbara, California 93106, USA, ${ }^{4}$ Department of Physics, Case Western Reserve University, Cleveland, Ohio 44106, USA. *e-mail: jonschuller@ece.ucsb.edu; rashid_zia@brown.edu 
a
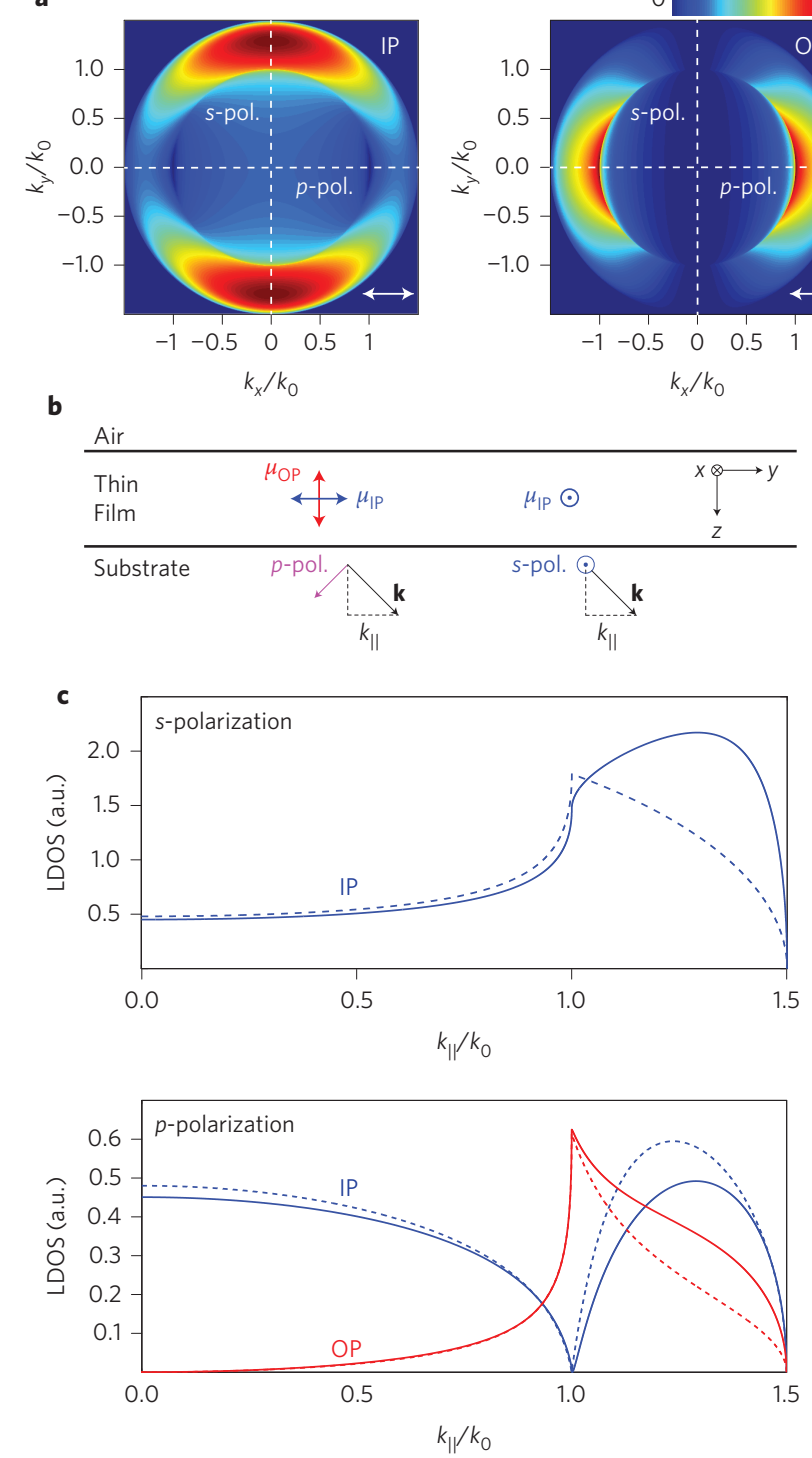

Figure 1 | Theory of IP and OP dipole emission. a, Calculated $x$-polarized back focal plane emission patterns from purely IP (left) and OP (right) dipoles emitting at $700 \mathrm{~nm}$, located in the middle of a 20-nm-thin uniaxial layer of PTCDA with IP and OP refractive indices of $n_{\mathrm{o}}=2.1$ and $n_{\mathrm{e}}=1.6$, respectively, sitting on a quartz substrate $(n=1.5)$. The solid white arrows denote the $x$-oriented polarization direction. The $s$ - and $p$-polarized crosssections are indicated by white dashed lines. $\mathbf{b}$, Schematic of the layered geometry, highlighting the relationship between dipole orientations ( $\mu_{\mathrm{IP}}$ and $\left.\mu_{\mathrm{OP}}\right)$ and emission polarization. c, The s- (top) and $p$ - (bottom) polarized LDOS for IP (blue) and OP (red) dipoles. Solid lines: the 20-nm-thin film of PTCDA considered in $\mathbf{a}$. Dashed lines: an infinitesimally thin film using the electrostatic approximations derived in Supplementary Section IV. The $s$-polarized light only comes from IP dipoles. For $p$-polarized light, the IP LDOS vanishes at $k_{\|}=k_{0}$ but the OP LDOS is maximized.

investigating exciton orientations. The solid lines in Fig. 2a show the experimental momentum-resolved $s$ - (blue) and $p$ - (purple) polarized photoluminescence of monolayer $\mathrm{MoS}_{2}$ from the A exciton, which is associated with near band-edge direct-gap transitions at the $K$ and $K^{\prime}$ points of the Brillouin zone, modified by strong electron-hole interactions. The luminescence at $k_{\|}=k_{0}$ nearly vanishes, indicating a purely IP dipole moment. By fitting the $p$-polarized data (purple dashed line) at every wavelength, we derive a theoretical curve for $s$-polarization that is free of any fit parameters (blue
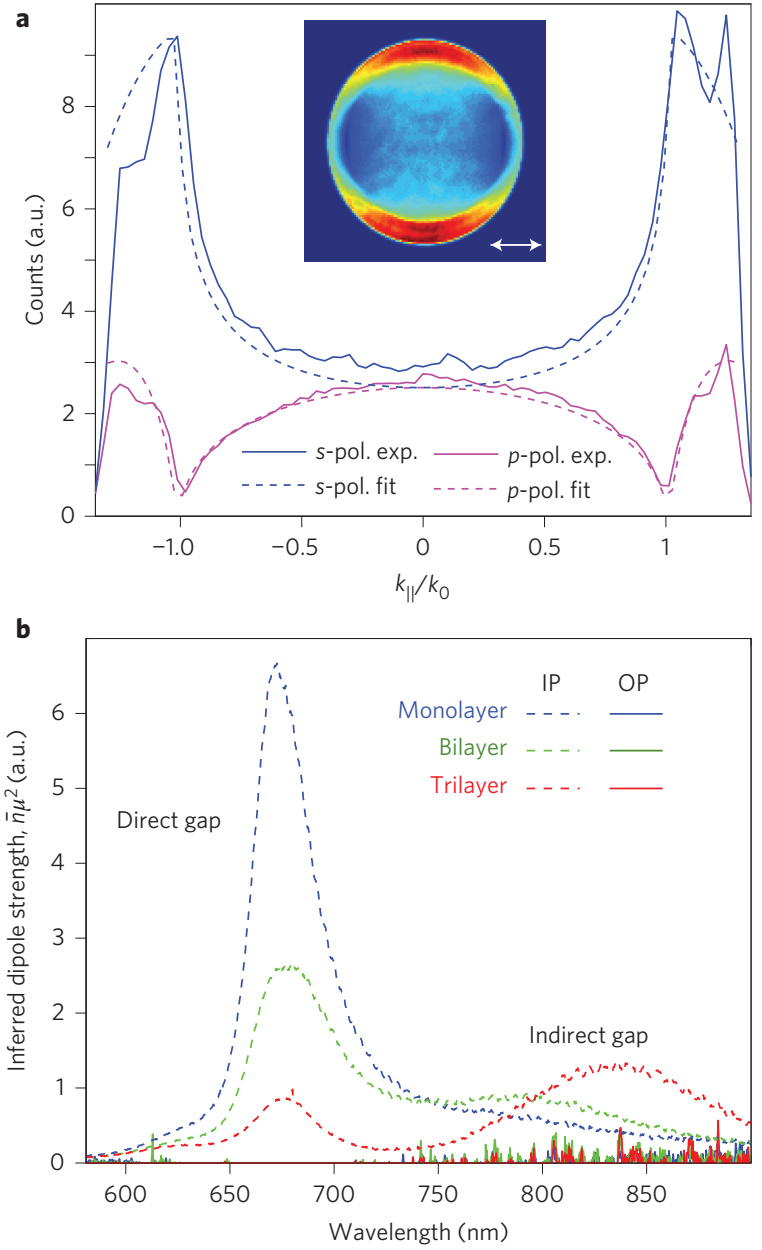

Figure 2 | Exciton orientation in mono-, bi- and trilayer $\mathrm{MoS}_{2}$.

a, Experimental momentum spectra of $664 \mathrm{~nm}$ photoluminescence from a monolayer of $\mathrm{MoS}_{2}$. Inset: the full $x$-polarized back focal plane image, which closely resembles the calculated IP back focal plane image shown in Fig. 1a. Solid lines: experimental s- (blue) and $p$ - (purple) polarized cross-sections at $664 \mathrm{~nm}$. The $p$-polarized cross-sections exhibit vanishing luminescence intensity at $k_{\|}=k_{0}$. The $p$-polarized data are fit (dashed lines) to a superposition of IP and OP dipole emission, generating an expected s-polarized spectrum that has no free fit parameters. b, Total OP (solid lines) and IP (dashed lines) dipole strengths inferred from independent fits to angular spectra at each wavelength. Both the direct $(\sim 660 \mathrm{~nm})$ and indirect ( $840 \mathrm{~nm}$ ) gap luminescence from $\mathrm{MoS}_{2}$ multilayers arise entirely from IP (intralayer)-oriented excitons.

dashed line) and determine the dipole strengths of the IP and OP excitons (Fig. 2b). We see excellent agreement between theory and experiment, and at every wavelength the data are best fit by assuming the photoluminescence arises entirely from IP excitons. Calculations ${ }^{18}$ and measurements ${ }^{4}$ indicate that the lowest energy optical dipole transitions in bulk $\mathrm{MoS}_{2}$ are purely IP. Because exciton relaxation is negligible, the direct-gap photoluminescence transitions are identical to those for near band-edge absorption. Thus, our results are consistent with previous studies and validate the experimental technique.

In multilayer $\mathrm{MoS}_{2}$, interlayer interactions modify the electronic band structure, suppressing direct-gap emission (hot photoluminescence) and giving rise to prominent lower-energy, indirect-gap photoluminescence. The indirect-gap feature in the photoluminescence spectra has been used to distinguish the number of layers in few-layer $\mathrm{MoS}_{2}$ (refs 16,17). However, the 

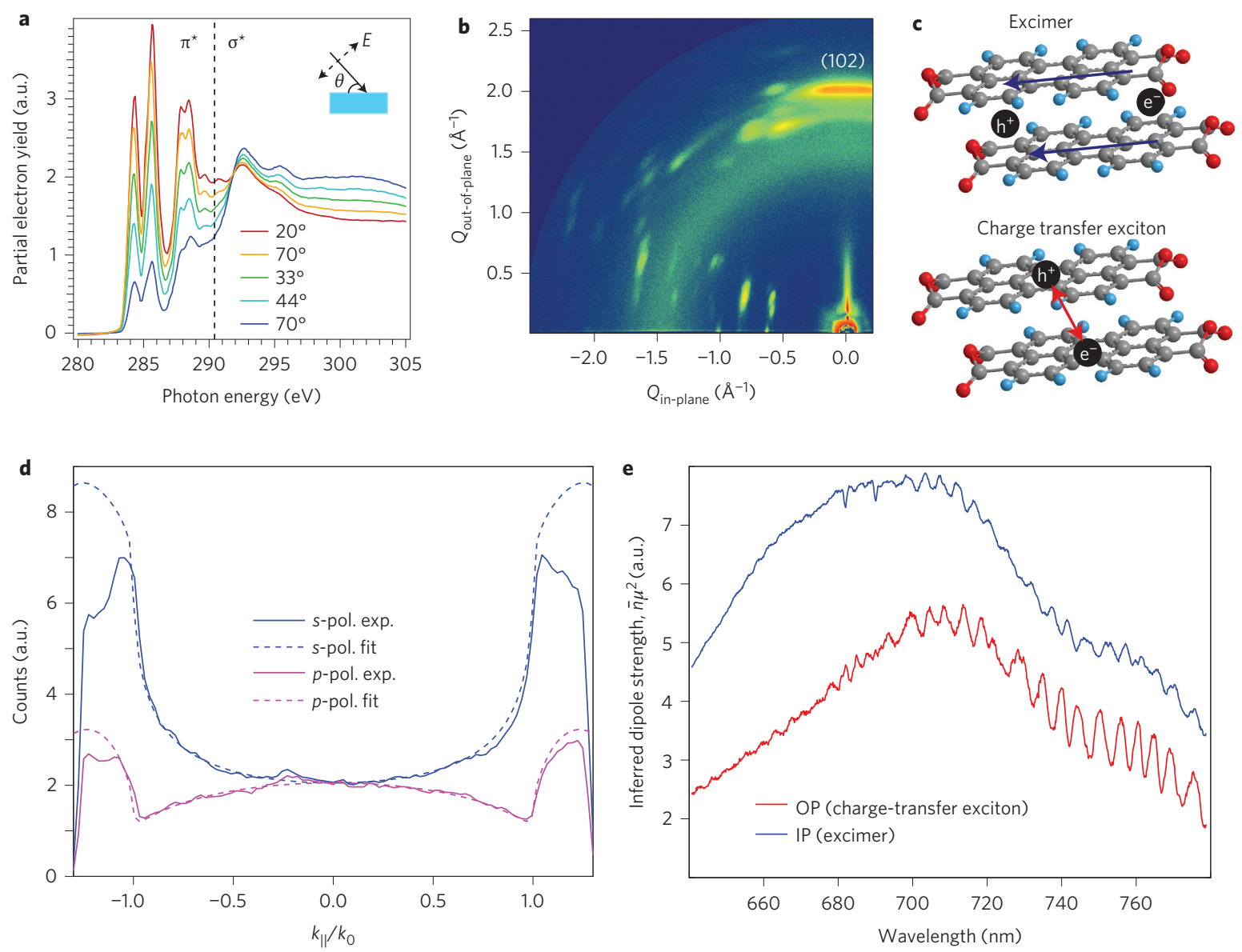

Figure 3 | Morphology and exciton orientations in a PTCDA thin film. a, Angle-dependent NEXAFS. The C $1 s \rightarrow \pi^{\star}$ transitions near $285 \mathrm{eV}$ and $\mathrm{C} 1 \mathrm{~s} \rightarrow \sigma^{\star}$ transitions near $295 \mathrm{eV}$ both exhibit very strong dependence on the incident angle of the p-polarized X-ray source, indicating molecules that are highly oriented parallel to the sample surface. The two lowest-energy $\pi^{\star}$ peaks originate from carbon atoms in the aromatic perylene core, and the highest-energy $\pi^{\star}$ doublet originates from carbon atoms in anhydride functional groups. b, GIXD 2D reciprocal space patterns where colour corresponds to a logarithmic intensity scale. Diffraction peaks are consistent with a mixture of $\alpha$ - and $\beta$-phase PTCDA crystals (Supplementary Section III). The highest-intensity peak arises from (102) diffraction and is largely confined to the OP axis. This indicates a crystal orientation with molecules aligned parallel to the substrate surface, consistent with the NEXAFS results and previous structural investigations of PTCDA thin films. c, Schematics showing the orientation of excimers and charge transfer excitons relative to a pair of PTCDA molecules within the crystalline thin film. $\mathbf{d}$, Solid lines: experimental s- (blue) and p-(purple) polarized momentum-resolved photoluminescence from a PTCDA thin film at $700 \mathrm{~nm}$. Dashed lines: associated fits. The luminescence intensity at $k_{\|}=k_{0}$ is nearly equal to that at $k_{\|}=0$. e, Inferred dipole strengths for IP and OP excitons in PTCDA. Oscillations with wavelength arise from etalon effects in the CCD array at long wavelengths. IP and OP emissions are assigned to excimers and interlayer charge transfer excitons, respectively.

orientation of the indirect-gap dipole moment is unknown, because this transition contributes negligibly to optical absorption. In general, photoluminescence measurements can reveal the properties of relaxed exciton states that are not probed by other methods. Our results (Fig. 2b) demonstrate that the indirect-gap transition is also IP-oriented. Even though this feature is only prominent in $\mathrm{MoS}_{2}$ multilayers, it is not the result of an OP interlayer transition. In the next section, we investigate a material in which strong interlayer coupling causes photoluminescence from both IP- and OP-oriented excitons.

Polycyclic aromatic hydrocarbons (PAHs) - such as pentacene, perylene and graphene-are tremendously important materials in contemporary organic electronics research. In PAHs, overlapping $p_{z}$ atomic orbitals form $\pi$ bonds with a delocalized electron density above and below the plane of the carbon atoms. In single PAH molecules, optical transitions between the highest-energy occupied molecular orbital $(\pi)$ and the lowest-energy unoccupied molecular orbital $\left(\pi^{\star}\right)$ are characterized by an IP dipole moment ${ }^{19}$. Here, we study photoluminescence from highly oriented thin films of the perylene derivative PTCDA.
We determined the molecular and crystallographic orientation of PTCDA films using angle-resolved near-edge X-ray absorption fine structure (NEXAFS) spectroscopy and grazing incidence X-ray diffraction (GIXD), respectively. The strong angular dependence of the $\mathrm{C} 1 s \rightarrow \pi^{*}$ transitions near $285 \mathrm{eV}$ (Fig. 3a) indicates molecules that are highly oriented parallel to the substrate (IP). GIXD patterns (Fig. $3 \mathrm{~b}$ ) show oriented crystals of both $\alpha$ - and $\beta$-PTCDA, with molecules preferentially oriented parallel to the substrate (Supplementary Section III, Figs S3 and S4). The high-intensity (102) diffraction peak near $2 \AA^{-1}$ arises from PTCDA molecules that are closely stacked ( $3.2 \AA)$ along the substrate normal (OP), resulting in a large overlap of $\pi$ orbitals and strong interlayer interactions $^{20}$. As a result of these interactions, the photoluminescence from PTCDA changes markedly as the material evolves from discrete single molecules to a layered nanomaterial ${ }^{21}$. In a weakly coupled material, the optical properties would be dominated by Frenkel excitons, which retain the localized orbitals and IP orientation characteristic of monomers. In PTCDA, however, theoretical models of both dimers ${ }^{22}$ and one-dimensional stacks ${ }^{23}$ capture the importance of the OP-oriented interlayer charge-transfer 


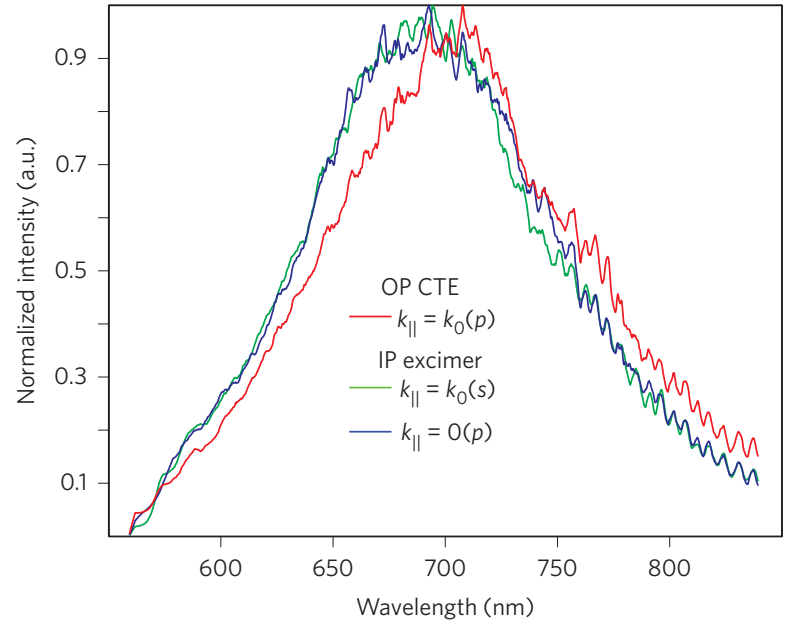

Figure 4 | Fit-independent method for isolating different exciton orientations. Normalized photoluminescence spectra from PTCDA for different momenta and polarizations. For s-polarization at $k_{\|}=k_{0}$ (green) and $p$-polarization at $k_{\|}=0$ (blue) the contribution from OP charge-transfer excitons (CTE) vanishes and the luminescence is purely from IP excimers. The reverse is true for $p$-polarized spectra at $k_{\|}=k_{0}$ (red). The observed frequency shift agrees with the results presented in Fig. 3e, but requires no fitting, modelling or knowledge of the sample thickness or refractive index. Note the difference in wavelength scale compared with Fig. 3e.

exciton-an electron and hole localized on adjacent molecular layers. The description of exciton states in PTCDA is further complicated by the existence of mixed charge transfer and Frenkel exciton states and IP-oriented intralayer charge-transfer excitons. Regardless of specific assignments, previous ellipsometry ${ }^{5}$ and electron energy loss spectroscopy (EELS) ${ }^{24}$ experiments indicate that our pump laser predominantly excites IP excitons.

After excitation, IP excitons in PTCDA films quickly undergo intraband relaxation to lower-energy Bloch states ${ }^{25}$ and lower their energy by coupling to interlayer lattice deformations ${ }^{26}$, a process referred to as self-trapping or excimer formation. Timeand temperature-dependent PTCDA photoluminescence spectra have previously been decomposed into contributions from different excitons using multi-Gaussian fits motivated by theoretical calculations ${ }^{27-29}$. These studies suggest that room-temperature photoluminescence arises primarily from IP excimers, with a small contribution from OP charge-transfer excitons that emit at nearly the same frequency.

The momentum-resolved photoluminescence from a PTCDA thin film is shown in Fig. 3d. Unlike $\mathrm{MoS}_{2}$, there is significant $p$-polarized (purple) luminescence at $k_{\|}=k_{0}$, indicating the presence of $\mathrm{OP}$ excitons. By fitting the photoluminescence traces (dashed lines), we determine relative measures of the intrinsic IP and OP dipole strengths $\bar{n}_{\mathrm{IP}}\left|\mu_{\mathrm{IP}}(\omega)\right|^{2}$ and $\bar{n}_{\mathrm{OP}}\left|\mu_{\mathrm{OP}}(\omega)\right|^{2}$ respectively (Fig. 3e). Although light absorption predominantly generates IP excitons, the dipole strengths for IP (blue) and OP (red) excitons are within a factor of two of one another. Additionally, the emission from OP excitons is redshifted compared with IP states. These results demonstrate significant differences between photoluminescence and absorption anisotropies. By identifying IP orientation with excimers and OP orientation with charge-transfer excitons (Fig. 3c), comparisons can be made with previous studies of PTCDA luminescence, where the contributions of various exciton species were determined by means of multi-Gaussian fits ${ }^{27-29}$. Both the large dipole strength and noticeable redshift of the OP charge-transfer exciton are not evident in these investigations, highlighting the importance of momentum-resolved techniques for experimentally distinguishing excitons by their orientation.

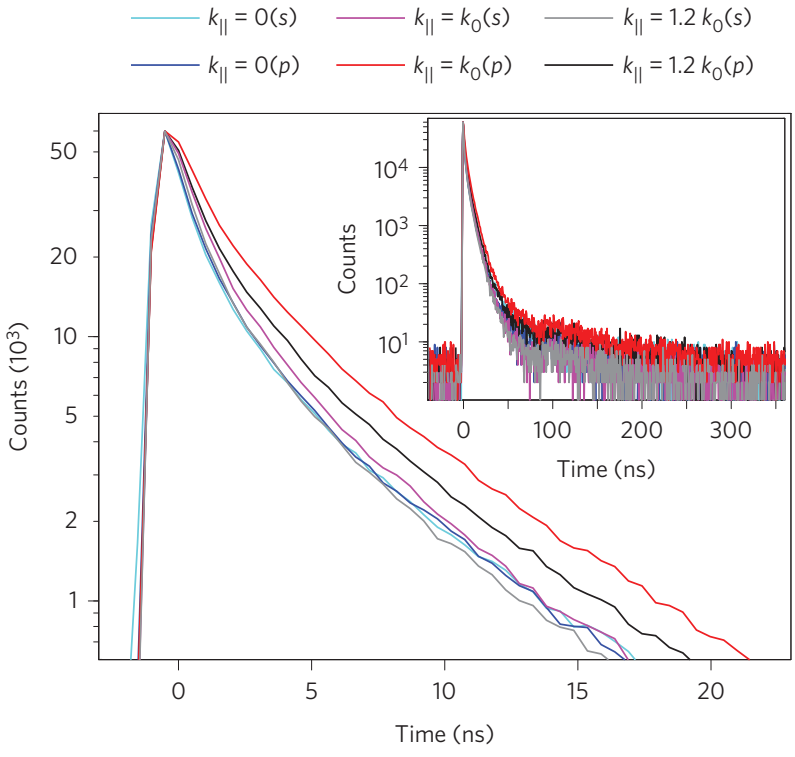

Figure 5 | Comparison of charge-transfer exciton and excimer dynamics. Semilogarithmic decay traces for $s$ - and $p$-polarized photoluminescence at various momentum values. All traces exhibit the same peak value of counts $(60,000)$ in the same time bin, which we define as $t=0$. The $s$-polarized photoluminescence (cyan, magenta, grey) and $p$-polarized photoluminescence at $k_{\|}=0$ (blue) arise only from IP excimers and have similar fast decays. The $p$-polarized photoluminescence at $k_{\|}=k_{0}$ (red) arises only from OP charge-transfer excitons and exhibits a significant shift to longer times indicative of slower dynamics. The slopes of the decay traces at late times are approximately equal; the largest difference in dynamics occurs at early times. The $p$-polarized photoluminescence at $k_{\|}=1.2 k_{0}$ (black) arises from both exciton species and exhibits intermediate dynamics.

As described previously, the thin-film LDOS for IP and OP emitters are significantly different at the onset of total internal reflection $\left(k_{\|}=k_{0}\right)$. At this momentum, $p$-polarized photoluminescence originates purely from OP excitons. However, the $p$-polarized luminescence at $k_{\|}=0$ and $s$-polarized luminescence at all momenta arise from IP excitons only. Thus, by filtering polarized luminescence in momentum space, we can obtain a fit-independent method for isolating photoluminescence from IP or OP excitons. Using an optical fibre as described in the Methods, we collected and plotted (Fig. 4) the normalized $s$-polarized spectrum at $k_{\|}=k_{0}$ (green) and the $p$-polarized spectra at $k_{\|}=0$ (blue) and $k_{\|}=k_{0}$ (red). The green and blue spectra are identical and representative of pure IP excimer emission. In contrast, the red spectrum arises from $\mathrm{OP}$ charge-transfer excitons only and exhibits a redshift similar to that shown in Fig. 3e (note the different wavelength scale). In addition to confirming our fit results, this technique enables more sophisticated experimental investigations.

By isolating the photoluminescence from different exciton orientations, we can use a single high-speed photodetector to independently study the dynamics of charge-transfer excitons and excimers (see Methods). Traces of time-resolved photoluminescence at various momenta and polarizations are shown in Fig. 5. These were taken at low pump fluence, where the dynamics are independent of pump fluence. All traces exhibit a fast decay component at early times and a slow decay component at later times ( $t>5 \mathrm{~ns}$ ). However, differences between traces demonstrate the existence of distinct exciton populations. All traces arising from IP emission (that is, the $p$-polarized trace at $k_{\|}=0$ (blue) and all $s$-polarized traces; cyan, magenta, grey) have identical decays and represent excimer photoluminescence. In contrast, the purely OP emission (that is, the $p$-polarized trace at $k_{\|}=k_{0}$ (red)) arises from 
charge-transfer excitons and has significantly slower dynamics, as evidenced by its separation from all other curves. The $p$-polarized trace at $k_{\|}=1.2 k_{0}$ (black) displays intermediate dynamics, as expected from a mixture of excitons. The majority of the difference between charge-transfer exciton and excimer dynamics occurs at early times and may arise from the distinct recombination pathways for the two exciton species. Alternatively, charge-transfer exciton decay may be convolved with a rising charge-transfer exciton population density as initially excited IP excitons reorient in the OP direction. Although the exact dynamical mechanisms are not yet known, it is clear that the room-temperature photoluminescence from PTCDA arises from two distinct exciton species with different spectra, dipole strengths and temporal dynamics.

Our results demonstrate the fundamental relationship between the morphology of thin-film materials and the orientation of excitons. Although the momentum-resolved techniques demonstrated here are restricted to thin films, they can be applied to any material and adapted to a variety of other optical measurements. For instance, although we have used momentum-resolved spectroscopies to isolate luminescence from IP and OP excitons, time-reversal symmetry dictates that these techniques can also be used to selectively excite either IP or OP excitons. Thus, the concepts and techniques presented here may lead to better control and understanding of both the generation and recombination processes in other highly oriented materials. In a planar bilayer heterojunction ${ }^{30}$ organic photovoltaic, for example, selective excitation of OP excitons could be used to directly measure the cross-section and energy spectrum of charge-transfer excitons at a donor/acceptor interface. Similarly, selective detection could be used to measure chargetransfer exciton dynamics associated with diffusion, dissociation and recombination processes. We anticipate these results will have a significant impact on both fundamental and applied organic optoelectronics research in a variety of materials systems ${ }^{1,31-34}$.

\section{Methods}

Experimental set-up. A schematic of the experimental apparatus is shown in Supplementary Fig. S2. The photoluminescence from our samples was excited and collected by an oil immersion objective (Nikon CFI-Plan Fluor $\times 100$, NA $=1.3$ ) mounted in an inverted Nikon Eclipse microscope. A Bertrand lens with a focal length of $50 \mathrm{~mm}$ was used to reimage the objective's back focal plane onto the entrance slit of an imaging spectrograph (Princeton Instruments SP2300i) equipped with a 150 lines $\mathrm{mm}^{-1}$ grating blazed at $800 \mathrm{~nm}\left(\mathrm{MoS}_{2}\right.$ data, Fig. 2) and a 300 lines $\mathrm{mm}^{-1}$ grating blazed at $500 \mathrm{~nm}$ (PTCDA data, Fig. 3). In imaging mode (Fig. 2a, inset), the slit was open wide, an angled mirror was used for specular reflection, and the back focal plane image was focused onto a Princeton Instruments Pixis 1024B charge coupled device (CCD) array. In spectroscopy mode (Figs 2 and 3 ), the slit was narrowed and the grating angled to disperse the photoluminescence by wavelength such that one axis of the CCD array corresponded to wavelength and the other to in-plane momentum. $\mathrm{MoS}_{2}$ data were taken with a $488 \mathrm{~nm}$ argon ion excitation laser (Coherent Innova 300C) and Princeton Instruments ProEM Excelon camera with a pixel width corresponding to $k_{\|} \approx 0.033 k_{0}$. PTCDA data were taken with a $532 \mathrm{~nm}$ excitation laser (Coherent Verdi) and Princeton Instruments Pixis $1024 \mathrm{~B}$ camera with a pixel width corresponding to $\Delta k_{\|} \approx 0.025 k_{0}$. The observed photoluminescence results were insensitive to the polarization of the pump laser for all measurements. All spectra presented are background-subtracted raw data without intensity calibration. The combination of chromatic dispersion in the objective, gratings and cameras causes a continual decrease in collection efficiency as the wavelength increases past $600 \mathrm{~nm}$. However, this does not affect the momentum-space fits, which are performed separately at each wavelength. Furthermore, the momentumspace fits were performed over the momentum range $-1.1 k_{0} \leq k_{\|} \leq 1.1 k_{0}$ to minimize the effect of imaging artefacts observed at higher-numerical-aperture values and to account for the lower effective numerical aperture of the coupled microscope-lens-spectrograph system ${ }^{15}$

For momentum filtering experiments, a Bertrand lens with a focal length of $250 \mathrm{~mm}$ was used to reimage the back focal plane to a much larger size. For spectroscopic measurements, the photoluminescence was filtered in momentum space by a $100 \mu \mathrm{m}$ (corresponding to $k_{\|} \approx 0.02 k_{0}$ ) core optical fibre coupled to the spectrograph/camera system described above, operating with a 150 lines $\mathrm{mm}^{-1}$ grating blazed at $500 \mathrm{~nm}$. For time-resolved measurements, a 100 - $\mu \mathrm{m}$-diameter $\left(k_{\|} \approx 0.02 k_{0}\right)$ circular pinhole on a translation stage was used to filter the photoluminescence, which was then reimaged onto the active area of a single photon avalanche diode (PicoQuant Tau-SPAD). Samples were excited by a $485 \mathrm{~nm}$ pulsed laser diode with sub-100 ps pulse widths at a $2.5 \mathrm{MHz}$ repetition rate (PicoQuant LDH-D-C-485). Signals from the photodiode were recorded with a resolution of 512 ps using a PicoHarp 300 time-correlated single-photon counting system. Photons were counted until a peak value of 60,000 was achieved. All traces exhibited a maximum at the same time bin. Traces were recorded at various pump powers to confirm that the decays were pump power-independent.

Sample fabrication. Few-layer $\mathrm{MoS}_{2}$ samples were mechanically exfoliated from bulk $\mathrm{MoS}_{2}$ crystals (SPI Supplies) and deposited on thin (150-180 $\left.\mu \mathrm{m}\right)$ quartz coverslips. Mono-, bi- and trilayer $\mathrm{MoS}_{2}$ were identified using optical microscopy. Their thicknesses were confirmed by atomic force microscopy (AFM) and photoluminescence measurements ${ }^{16}$. PTCDA films were thermally evaporated in a physical vapour deposition system at $<5 \times 10^{-7}$ torr with substrates held at room temperature. Films were deposited on quartz coverslips for photoluminescence measurements and on silicon substrates with a 100-nm oxide layer for reference AFM, ellipsometry and X-ray studies. Samples were annealed on a hot plate at $100{ }^{\circ} \mathrm{C}$ for $24 \mathrm{~h}$. To minimize free parameters, we determined the thickness of the PTCDA layers from AFM measurements $(20 \mathrm{~nm})$ and the refractive indices from ellipsometry measurements.

Received 20 August 2012; accepted 21 January 2013; published online 3 March 2013

\section{References}

1. DeLongchamp, D. M. et al. High carrier mobility polythiophene thin films: structure determination by experiment and theory. Adv. Mater. 19, 833-837 (2007).

2. Kim, D. W., Kim, Y. H., Jeong, H. S. \& Jung, H-T. Direct visualization of largearea graphene domains and boundaries by optical birefringency. Nature Nanotech. 7, 29-34 (2011).

3. Greenaway, D. L., Harbeke, G., Bassani, F. \& Tosatti, E. Anisotropy of the optical constants and the band structure of graphite. Phys. Rev. 178, 1340-1348 (1969).

4. Liang, W. Optical anisotropy in layer compounds. J. Phys. C 6, 551-565 (1973).

5. Alonso, M. I., Garriga, M., Karl, N., Ossó, J. O. \& Schreiber, F. Anisotropic optical properties of single crystalline PTCDA studied by spectroscopic ellipsometry. Org. Electron. 3, 23-31 (2002).

6. Najafov, H., Lee, B., Zhou, Q., Feldman, L. C. \& Podzorov, V. Observation of long-range exciton diffusion in highly ordered organic semiconductors. Nature Mater. 9, 938-943 (2010).

7. Wasey, J. A. \& Barnes, W. Birefringence and light emission from the polymer LED. Synthetic Met. 111, 213-215 (2000).

8. Böhmler, B. et al. A. Enhancing and redirecting carbon nanotube photoluminescence by an optical antenna. Opt. Express 18, 16443-16451 (2010).

9. Chuang, S-Y., Yu, C-C., Chen, H-L., Su, W-F. \& Chen, C-W. Exploiting optical anisotropy to increase the external quantum efficiency of flexible P3HT:PCBM blend solar cells at large incident angles. Sol. Energ. Mat. Sol. C 95, 1-10 (2011).

10. Neale, S. L., MacDonald, M. P., Dholakia, K. \& Krauss, T. F. All-optical control of microfluidic components using form birefringence. Nature Mater. 4, 530-533 (2005)

11. Hoffman, A. J. et al. Negative refraction in semiconductor metamaterials. Nature Mater. 6, 946-950 (2007).

12. Lieb, M. A., Zavislan, J. M. \& Novotny, L. Single-molecule orientations determined by direct emission pattern imaging. J. Opt. Soc. Am. B 21, 1210-1215 (2004).

13. Lieberherr, M., Fattinger, Ch. \& Lukosz, W. Optical-environment-dependent effects on the fluorescence of submonomolecular dye layers on interfaces. Surf. Sci. 189-190, 954-959 (1987).

14. Curto, A. G. et al. Unidirectional emission of a quantum dot coupled to a nanoantenna. Science 329, 930-933 (2010).

15. Taminiau, T. H., Karaveli, S., van Hulst, N. F. \& Zia, R. Quantifying the magnetic nature of light emission. Nature Commun. 3, 979 (2012).

16. Mak, K. F., Lee, C., Hone, J., Shan, J. \& Heinz, T. F. Atomically thin $\mathrm{MoS}_{2}$ : a new direct-gap semiconductor. Phys. Rev. Lett. 105, 136805 (2010).

17. Splendiani, A. et al. Emerging photoluminescence in monolayer $\mathrm{MoS}_{2}$. Nano Lett. 10, 1271-1275 (2010).

18. Reshak, A. \& Auluck, S. Calculated optical properties of $2 \mathrm{H}-\mathrm{MoS}_{2}$ intercalated with lithium. Phys. Rev. B 68, 1-7 (2003).

19. Pope, M. \& Swenberg, C. E. Electronic Processes in Organic Crystals and Polymers (Oxford Univ. Press, 1999).

20. Forrest, S. R. Ultrathin organic films grown by organic molecular beam deposition and related techniques. Chem. Rev. 97, 1793-1896 (1997).

21. Proehl, H., Dienel, T., Nitsche, R. \& Fritz, T. Formation of solid-state excitons in ultrathin crystalline films of PTCDA: from single molecules to molecular stacks. Phys. Rev. Lett. 93, 097403 (2004).

22. Hennessy, M. H., Soos, Z. G., Pascal, R. A. \& Girlando, A. Vibronic structure of PTCDA stacks: the exciton-phonon-charge-transfer dimer. Chem. Phys. 245, 199-212 (1999).

23. Hoffmann, M. et al. The lowest energy Frenkel and charge-transfer excitons in quasi-one-dimensional structures: application to MePTCDI and PTCDA crystals. Chem. Phys. 258, 73-96 (2000). 
24. Knupfer, M., Schwieger, T., Fink, J., Leo, K. \& Hoffmann, M. Excitons in quasione-dimensional organic crystals. Phys. Rev. B 66, 035208 (2002).

25. Engel, E., Koschorreck, M., Leo, K. \& Hoffmann, M. Ultrafast relaxation in quasi-one-dimensional organic molecular crystals. Phys. Rev. Lett. 95, 157403 (2005).

26. Scholz, R., Kobitski, A. Y., Zahn, D. R. T. \& Schreiber, M. Investigation of molecular dimers in $\alpha$-PTCDA by ab initio methods: binding energies, gas-tocrystal shift, and self-trapped excitons. Phys. Rev. B 72, 245208 (2005).

27. Kobitski, A. Y., Scholz, R., Zahn, D. R. \& Wagner, H. P. Time-resolved photoluminescence study of excitons in $\alpha$-PTCDA as a function of temperature. Phys. Rev. B 68, 155201 (2003).

28. Wagner, H. P., DeSilva, A. \& Kampen, T. Exciton emission in PTCDA films and PTCDA/Alq ${ }_{3}$ multilayers. Phys. Rev. B 70, 235201 (2004).

29. Gangilenka, V. R. et al. Exciton emission in PTCDA thin films under uniaxial pressure. Phys. Rev. B 77, 115206 (2008).

30. Tada, A., Geng, Y., Wei, Q., Hashimoto, K. \& Tajima, K. Tailoring organic heterojunction interfaces in bilayer polymer photovoltaic devices. Nature Mater. 10, 450-455 (2011).

31. Campoy-Quiles, M., Etchegoin, P. G. \& Bradley, D. D. C. On the optical anisotropy of conjugated polymer thin films. Phys. Rev. B 72, 045209 (2005).

32. Gurau, M. C. et al. Measuring molecular order in poly(3-alkylthiophene) thin films with polarizing spectroscopies. Langmuir 23, 834-842 (2007).

33. Dressel, M. et al. Kramers-Kronig-consistent optical functions of anisotropic crystals: generalized spectroscopic ellipsometry on pentacene. Opt. Express 16, 19770-19778 (2008).

34. Gordan, O. D., Friedrich, M. \& Zahn, D. R. T. The anisotropic dielectric function for copper phthalocyanine thin films. Org. Electron. 5, 291-297 (2004).

\section{Acknowledgements}

The authors thank S. Cueff, C. Dodson, T.F. Heinz, M. Jiang, J.A. Kash, J.A. Kurvits, D. Li, K.F. Mak, T.H. Taminiau and J.T. Yardley for helpful discussions. Support for R.Z. and S.K., travel for J.A.S., and the optical experiments were provided by the Air Force Office of Scientific Research (PECASE award no. FA-9550-10-1-0026), the National Science Foundation (CAREER award no. EECS-0846466, MRSEC award no. DMR-0520651) and the Nanoelectronic Research Initiative of the Semiconductor Research Corporation. J.A.S., T.S., S.Y. and I.K. were supported as part of the Center for Re-Defining Photovoltaic Efficiency through Molecule Scale Control, an Energy Frontier Research Center funded by the US Department of Energy, Office of Science, Office of Basic Energy Sciences (award no. DE-SC0001085). K.H. and J.S. were supported by the National Science Foundation (DMR-0907477). GIXD measurements were carried out at beamline 11-3 at the Stanford Synchrotron Radiation Light Source, SLAC National Accelerator Laboratory, a national user facility operated by Stanford University on behalf of the US Department of Energy, Office of Basic Energy Sciences. NEXAFS measurements were carried out at beamline U7A at the National Synchrotron Light Source, Brookhaven National Laboratory, which is supported by the US Department of Energy, Office of Basic Energy Sciences (contract no. DE-AC02-98CH10886)

\section{Author contributions}

J.A.S., S.K. and R.Z. designed and performed the optical experiments. T.S. performed and analysed GIXD and NEXAFS measurements. K.H. and J.S. fabricated the $\mathrm{MoS}_{2}$ samples. S.Y. and I.K. fabricated the PTCDA samples. J.A.S., S.K. and R.Z. analysed the data and implemented the theoretical model. All authors provided detailed feedback on the results and helped write the manuscript.

\section{Additional information}

Supplementary information is available in the online version of the paper. Reprints and permissions information is available online at www.nature.com/reprints. Correspondence and requests for materials should be addressed to J.A.S. and R.Z.

\section{Competing financial interests}

The authors declare no competing financial interests. 